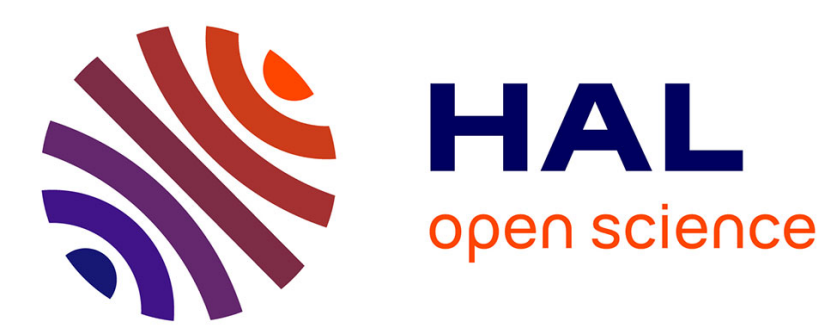

\title{
Impact of Antennas on the Anchor-less Indoor Localization of a Static IR-UWB Pair
}

Vincenzo La Tosa, Benoît Denis, Serge Bories, Bernard Uguen

\section{To cite this version:}

Vincenzo La Tosa, Benoît Denis, Serge Bories, Bernard Uguen. Impact of Antennas on the Anchorless Indoor Localization of a Static IR-UWB Pair. Personal Indoor and Mobile Radio Communications (PIMRC), 2010, Sep 2010, Istambul, Turkey. pp.858-864, 10.1109/PIMRC.2010.5672035 . hal-00684277

\section{HAL Id: hal-00684277 https://hal.science/hal-00684277}

Submitted on 31 Mar 2012

HAL is a multi-disciplinary open access archive for the deposit and dissemination of scientific research documents, whether they are published or not. The documents may come from teaching and research institutions in France or abroad, or from public or private research centers.
L'archive ouverte pluridisciplinaire $\mathbf{H A L}$, est destinée au dépôt et à la diffusion de documents scientifiques de niveau recherche, publiés ou non, émanant des établissements d'enseignement et de recherche français ou étrangers, des laboratoires publics ou privés. 


\section{Impact of Antennas on the Anchor-less Indoor Localization of a Static IR-UWB Pair}

\author{
V. La Tosa, B. Denis, S. Bories \\ CEA-Leti Minatec \\ 17 rue des Martyrs, 38054 Grenoble, France \\ \{vincenzo.latosa, benoit.denis, serge.bories\}@cea.fr
}

\author{
B. Uguen \\ IETR-CNRS, Université Rennes-I \\ Av. du Général Leclerc, 35042 Rennes, France \\ bernard.uguen@univ-rennes1.fr
}

\begin{abstract}
This paper investigates the impact of realistic antennas on joint anchor-less localization and indoor characterization based on Impulse Radio (IR) Ultra Wideband (UWB) communications. In this frame, the Maximum Averaged Likelihood (MAL) algorithm and its extended version are considered, both relying on a tree approach consisting in two stages. The first part of the process exploits the cross-correlation between received and locally predicted paths and it is the same for both algorithms. The second stage calculates the averaged likelihood of measured path parameters obtained in the previous step, but different measurements are used for MAL and extended MAL (eMAL), as in the first case only the Angle of Incidence (AoI) and the Time of Arrival (ToA) are considered. Additionally, the eMAL tree algorithm also accounts for two couples of Angles of Departure (AoDs) and Angles of Arrival (AoAs). The estimation errors of both nodes coordinates and room dimension obtained with the two algorithms are compared for three realistic UWB antennas. Finally, the remaining algorithm-independent ambiguities (i.e. resulting from scenario and geometry) are discussed.
\end{abstract}

Index Terms-Anchor-less Localization, Context-Awareness, Impulse Radio, Indoor Environments Characterization, Ultra Wide Band Antennas, Wireless Sensor Networks

\section{INTRODUCTION}

For the last years, new emerging needs have caused an increasing interest in Wireless Sensor Networks. Indeed, multiple applications have been conceived for future daily life, for instance dealing with automation in indoor environments (e.g. [1]). In this framework, the intrinsic fine temporal resolution and the low power consumption make the Impulse Radio (IR) - Ultra Wide Band (UWB) technology (e.g. [2]) a relevant solution for joint short-range communication and localization.

Indoor localization based on reference nodes (e.g. [3]) is one of the most successful and studied exploitation of IRUWB, but joint opportunistic anchor-less localization and basic indoor environment characterization have been also investigated. The two main contributions in this field are the Indoor Mapping technique (e.g. [4]) and a radar-like channel sounding method called Imaging (e.g. [5], [6]). In robotics, several solutions have been proposed for the Simultaneous Localization and Mapping (SLAM) problem (e.g. [7]), exploiting embedded cameras or laser rangers. Assuming a Conical Monopole Antenna (CMA) (e.g. [9]) at both transmitter and receiver, the previous work [8] presented the Maximum Averaged Likelihood (MAL) tree algorithm, exploiting Angles of Incidence (AoIs) (i.e. on the reflecting surface) and Times of Arrival (ToAs) (i.e. taken with respect to the receiver local reference) measurements.

This paper presents an extension of MAL algorithm and shows the results of anchor-less positioning and basic room characterization obtained with three realistic UWB antennas increasingly portable (i.e. with respect to dimensions and weight). This work has two main objectives: assessing if the knowledge of Angle of Departure (AoD) and Angle of Arrival (AoA) can improve MAL performances and understand the relationship between algorithms and antennas radiation diversity.

The paper is organized as follows. Section II describes the multipath model, the related assumptions and the chosen scenario. Section III shows the formalism of the averaged likelihood approach. Then, Section IV presents the proposed algorithms, while Section V discusses their limits and suggests some possible solutions and Section VI surveys the three real antennas. Finally, Section VII shows algorithm performances in both localization and basic environment characterization and Section VIII concludes the paper.

\section{Signal Model AND SCEnARIO}

In the context of IR-UWB indoor communications, the tension signal $\rho(t)$ at the receiver is generally described as a sum of paths, resulting from $N_{i n t}$ electromagnetic interactions:

$$
\rho(t)=\sum_{n=0}^{N_{\text {int }}} r_{n}\left(t-\tau_{n}\right)+\eta(t)
$$

where $r_{n}(t)$ is the $n$-th filtered received path, $\eta(t)$ is the noise affecting the communication filtered in the transmitted signal bandwidth and $\tau_{n}$ is the $n$-th path delay. The deterministic model described in [10] is assumed for a generic received tension in frequency domain $R(f)$. The generic path $r_{n}(t)$ can be obtained from $R(f)$ by an inverse Fourier transform. For the sake of simplicity, only single-bounce reflections in the 2D plane are considered and a Line Of Sight (LOS) channel is assumed. The simplified 2D indoor scenario is a rectangular room which walls are made of brick with a known thickness (e.g. $7 \mathrm{~cm})$. Therefore, referring to Figure 1, the largest room dimension is defined as $D_{x}$ and the transmitter is materialized as a grey spot while the receiver a dark grey spot, with coordinates $\left(x_{t}, y_{t}\right)$ and $\left(x_{r}, y_{r}\right)$ respectively.

\section{PATH PARAMETERS Model}

Four quantities will be associated to the $n$-th reflected path: AoD $\phi_{t, n}$, AoA $\phi_{r, n}$, AoI $\theta_{n}$ and ToA $\tau_{n}$, the latter taken with respect to a local time reference. As a general remark, note that variables numbering is the same as that of interacting walls numbering, where walls are arbitrary numbered anticlockwise. As an example, $\theta_{2}, \phi_{t, 2}, \phi_{r, 2}$ and $\tau_{2}$ are respectively the AoI, AoD, AoA and ToA of the path that has been reflected on wall 2 (i.e. the wall on the right on Figure 1). As the direct path is not associated with a wall interaction, the corresponding ToA will be noted as $\tau_{0}$.

As in [8], a Normal measurement error model will be assumed in the following for AoI $\theta_{n}$ and ToAs $\tau_{n}$, with standard deviations $\sigma_{\theta}$ and $\sigma_{\tau}$ respectively. Therefore, according to the independence assumption of measurements for each $n$ (e.g. 
as in [8]), the joint truncated Probability Density Function

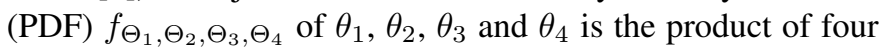
independent truncated Normal densities, as follows:

$$
f_{\Theta_{1}, \Theta_{2}, \Theta_{3}, \Theta_{4}}\left(\theta_{1}, \theta_{2}, \theta_{3}, \theta_{4}\right)=\prod_{n=1}^{4} f_{\Theta_{n}}\left(\theta_{n}\right)
$$

where

$$
f_{\Theta_{n}}\left(\theta_{n}\right)=\frac{\exp \left\{-\frac{1}{2}\left(\frac{\theta_{n}-\mu_{\theta_{n}}}{\sigma_{\theta}}\right)^{2}\right\}}{\operatorname{erf}\left(\frac{\frac{\pi}{2}-\mu_{\theta_{n}}}{\sqrt{2} \sigma_{\theta}}\right)-\operatorname{erf}\left(\frac{-\mu_{\theta_{n}}}{\sqrt{2} \sigma_{\theta}}\right)}
$$

and the normalization factor accounts for the truncation on interval $\left[0, \frac{\pi}{2}\right]$. Let us define the $n$-th ToA difference $\Delta \tau_{n}=$ $\tau_{n+1}-\tau_{n}$, then the joint PDF $f_{\Delta T_{0}, \Delta T_{1}, \Delta T_{2}, \Delta T_{3}}$ of differences $\Delta \tau_{0}, \Delta \tau_{1}, \Delta \tau_{2}$ and $\Delta \tau_{3}$ is described by:

$$
\begin{aligned}
& f_{\Delta T_{0}, \Delta T_{1}, \Delta T_{2}, \Delta T_{3}}\left(\Delta \tau_{0}, \Delta \tau_{1}, \Delta \tau_{2}, \Delta \tau_{3}\right)= \\
& \frac{1}{(2 \pi)^{2} \sqrt{5} \sigma_{\tau}} \exp \left\{-\frac{1}{2}\left[\underline{\Delta \tau}-\underline{\mu_{\Delta \tau}}\right]^{\mathrm{T}} \underline{\underline{D}}^{-1}\left[\underline{\Delta \tau}-\underline{\mu_{\Delta \tau}}\right]\right\}
\end{aligned}
$$

where $\underline{\underline{\mathrm{D}}}$ is the covariance matrix written as follows:

$$
\underline{\underline{D}}=\left[\begin{array}{cccc}
2 \sigma_{\tau}^{2} & \sigma_{\tau}^{2} & 0 & 0 \\
\sigma_{\tau}^{2} & 2 \sigma_{\tau}^{2} & \sigma_{\tau}^{2} & 0 \\
0 & \sigma_{\tau}^{2} & 2 \sigma_{\tau}^{2} & \sigma_{\tau}^{2} \\
0 & 0 & \sigma_{\tau}^{2} & 2 \sigma_{\tau}^{2}
\end{array}\right]
$$

Finally, like in [8], the noise affecting range measurements is assumed to have a standard deviation $\sigma_{d}=c \sigma_{\tau}$ and follows the Normal PDF $f_{D}(d)$ below:

$$
f_{D}(d)=\frac{1}{\sqrt{2 \pi} \sigma_{d}} \exp \left\{-\frac{1}{2}\left(\frac{d-\mu_{d}}{\sigma_{d}}\right)^{2}\right\}
$$

where $\mu_{d}=\sqrt{\left(x_{r}-x_{t}\right)^{2}+\left(y_{r}-y_{t}\right)^{2}}$ and $c$ is light velocity.

The extended algorithm also considers $\phi_{t, n}$ and $\phi_{r, n}$ measurements, in order to assess if their approximate knowledge can reduce anchor-less positioning error in comparison with what obtained with MAL and presented in [8]. Nevertheless, because of the unknown orientation of the peers, $\phi_{t, n}$ and $\phi_{r, n}$ can not be written as functions of peers coordinates. Indeed, the simple knowledge of directions $\phi_{t, n}$ and $\phi_{r, n}$ can not directly contribute to nodes anchor-less positioning although the difference of AoDs and AoAs does. In this context, AoDs differences will be called $\alpha$ and AoAs differences $\beta$. In the particular case of differences calculated with respect to direct path AoD $\phi_{t, 0}$ and AoA $\phi_{r, 0}$, the following quantities can be defined:

$$
\begin{aligned}
& \alpha_{n, 0}=\phi_{t, n}-\phi_{t, 0} \\
& \beta_{n, 0}=\phi_{r, n}-\phi_{r, 0}
\end{aligned}
$$

where $n \in\{1, \ldots, 4\}$. Therefore, using the simple geometrical equations linking $\alpha_{n, 0}$ and $\beta_{n, 0}$ to nodes coordinates and room dimension $D_{x}$ is expected to improve estimation performances when these measurements are considered in the likelihood approach. Moreover, measurements $\theta_{n}, \phi_{t, n}, \phi_{r, n}$ and $\tau_{n}$ will be considered independent from each other and for each $n$. Under the assumption of $\phi_{t, n}$ and $\phi_{r, n}$ as Normal independent measurements both characterized by standard deviations $\sigma_{\phi}$, the marginal PDFs $f_{A_{n, 0}}$ of $\alpha_{n, 0}$ and $f_{B_{n, 0}}$ of $\beta_{n, 0}$ can be simply written as follows:

$$
\begin{aligned}
& f_{A_{n, 0}}\left(\alpha_{n, 0}\right)=\frac{1}{\sqrt{\pi} 2 \sigma_{\phi}} \exp \left\{-\frac{1}{2}\left(\frac{\alpha_{n, 0}-\mu_{\alpha_{n, 0}}}{\sqrt{2} \sigma_{\phi}}\right)^{2}\right\} \\
& f_{B_{n, 0}}\left(\beta_{n, 0}\right)=\frac{1}{\sqrt{\pi} 2 \sigma_{\phi}} \exp \left\{-\frac{1}{2}\left(\frac{\beta_{n, 0}-\mu_{\beta_{n, 0}}}{\sqrt{2} \sigma_{\phi}}\right)^{2}\right\}
\end{aligned}
$$

As an example, true values $\mu_{\alpha_{1,0}}$ and $\mu_{\beta_{1,0}}$ of $\alpha_{1,0}$ and $\beta_{1,0}$ can be respectively written as follows:

$$
\begin{gathered}
\mu_{\alpha_{1,0}}=\arcsin \left(2 \frac{y_{r}}{d} \sin \left(\arctan \left(\left|\frac{x_{r}-x_{t}}{y_{r}+y_{t}}\right|\right)\right)\right) \\
\mu_{\beta_{1,0}}=\arcsin \left(2 \frac{y_{t}}{d} \sin \left(\arctan \left(\left|\frac{y_{t}-y_{r}}{x_{t}+x_{r}-2 D_{x}}\right|\right)\right)\right)
\end{gathered}
$$

showing explicitly the dependence of true values and nodes coordinates. An illustration of $\alpha_{1,0}$ and $\beta_{1,0}$ is shown on Figure 1.

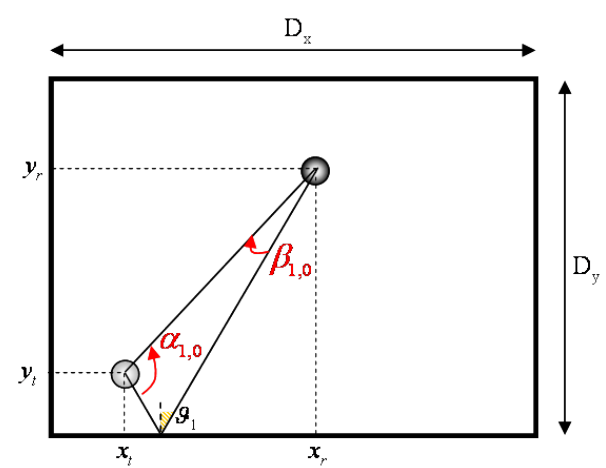

Fig. 1. Simplified multipath indoor 2D scenario

\section{Proposed Algorithms}

In the following two algorithms will be described and then compared by simulations, namely the MAL and a modified version called extended MAL (eMAL). Both algorithms rely on an estimation tree and use a path-by-path approach, which seems a natural choice given the intrinsic separability of IRUWB received signal. As a common prior stage, a database containing the expected received paths in the time domain is synthesized once and for all, according to the deterministic model described in [11]. Indeed, the AoI domain $\left[0, \frac{\pi}{2}\right]$ is considered as made up of $N_{\theta}$ clusters indexing the waveforms database, as in [11].

\section{A. Cross-correlation Stage}

The cross-correlation between successively detected paths and the synthesized waveforms of the database is calculated, in order to exploit the intrinsic radiation diversity of UWB antennas. This stage enables to identify a set of alike waveforms and provides, for each of them, the corresponding measurements $\phi_{t}, \phi_{r}, \theta$ and $\tau$. In order to reduce the overall complexity, the algorithm lets survive only the subset of the most correlated waveforms for each detected path. Like in [8], $\xi_{C C}$ represents the percentage of waveforms rejected because of their lowest cross-correlation value. The rejection percentage $0<\xi_{C C}<1$ is a priori set according to the observed deformation of the received waveforms generated by antennas.

1) MAL policy: For each detected path identified as a reflected path, only a quartet $\phi_{t}, \phi_{r}, \theta, \tau$ is chosen. Indeed, for a given AoI $\theta$, the chosen couple $\left(\phi_{t}, \phi_{r}\right)$ corresponds to the maximum calculated cross-correlation value. So, recalling that $N_{\theta}$ is the number of AoIs indexing the local database, the rejection factor $\xi_{C C}$ is applied to the $N_{\theta}$ chosen quartets.

2) eMAL policy: For each reflected path detected in the received signal, up to 3 possible quartets $\phi_{t}, \phi_{r}, \theta, \tau$ can be chosen. Given an AoI $\theta$, the couples of selected $\phi_{t}, \phi_{r}$ correspond to the $1 \%$ most correlated of waveforms, for which the mutual Euclidean distance in the 2D Cartesian system of coordinates $\left(\phi_{t}, \phi_{r}\right)$ is bigger than $\sqrt{2} \sigma_{\phi}$. Therefore, up to $3 N_{\theta}$ couples make up the set on which a rejection factor $\xi_{C C}$ is applied. 


\section{B. Averaged Likelihood Stage}

In order to limit the growth of the estimation tree, the path parameters surviving to the previous stage undergo a further rejection stage, based on the Maximum Averaged Likelihood.

Like in [8], when $n$ reflected paths has been detected at the $l$-th tree layer, the maximum $A L_{\max }$ of the Averaged Likelihood Function (ALF) $\Omega_{n}$ is evaluated for the $l$ quartets of path parameters available on the current tree branch. Note that $l=n+1$ in the LOS case and $l=n$ otherwise. Indeed, at the $l$-th tree layer, a branch corresponds to the first $l$ detected path parameters, as follows:

$$
\left\{\left(\phi_{t, j}, \phi_{r, j}, \theta_{j}, \tau_{j}\right): j \in\{1, \ldots, l\}\right\}
$$

In the following, according to the likelihood approach, these path parameters will be considered as measurements of true path parameters. Like in [8], $\Omega_{n}$ is the mixture of likelihood functions accounting for $n$ detected reflected paths and all possible labels mappings, weighting equally each possible label configuration. Indeed, the detection of a path does not on its own enable to estimate the path label (i.e. the numbering corresponding to the model described in Section III). By contrast, the mixture $\Omega_{n}$ is a solution to the label mapping ambiguity above, as the component corresponding to the right label mapping is expected to dominate remaining components.

Then, at each tree layer, a fixed number of candidates is eliminated because of their lowest $A L_{\max }$, with a rejection percentage $0<\delta_{L T, l}<1$. For instance, $\delta_{L T, l}=0.70$ means that, at the $l$-th layer, only the $30 \%$ of candidates having the highest values of $A L_{\max }$ (i.e. among these that passed the cross-correlation rejection stage) are enabled to join the $(l+1)$-th layer. Thus, all the surviving waveforms are treated in parallel, subtracted respectively from the received multipath signal when the first path has been detected and from the previous cleaned received signal otherwise. Therefore, at the end of this stage, $N_{\text {surv }, l}$ "cleaned" received signals and $N_{\text {surv }, l}$ sets of measurements $\phi_{t}, \phi_{r}, \theta$ and $\tau$ are available.

1) MAL policy: According to [8], only the subset made up of $N_{\text {surv }, l}$ measurements $(\theta, \tau)$ is considered. As an example of ALF, $\Omega_{1, L O S}$ (i.e. used when $n=1$ reflected path has been detected in addition to direct path) can be written as follows:

$$
\begin{aligned}
\Omega_{1, L O S}=\frac{1}{4} f_{D}(d)( & f_{\Theta_{1}}\left(\theta^{(1)}\right) f_{\Delta T_{1,0}}\left(\Delta \tau^{(1,0)}\right)+ \\
& f_{\Theta_{2}}\left(\theta^{(1)}\right) f_{\Delta T_{2,0}}\left(\Delta \tau^{(1,0)}\right)+ \\
& f_{\Theta_{3}}\left(\theta^{(1)}\right) f_{\Delta T_{3,0}}\left(\Delta \tau^{(1,0)}\right)+ \\
& \left.f_{\Theta_{4}}\left(\theta^{(1)}\right) f_{\Delta T_{4,0}}\left(\Delta \tau^{(1,0)}\right)\right)
\end{aligned}
$$

where $\Delta \tau^{(u, v)}$ is the ToA difference between the $u$-th received path ToA $\tau^{(u)}$ and the $v$-th received path ToA $\tau^{(v)}$, with a PDF $f_{\Delta T_{u, v}}\left(\Delta \tau^{(u, v)}\right)$ defined as follows:

$$
f_{\Delta T_{u, v}}\left(\Delta \tau^{(u, v)}\right)=\frac{1}{2 \sqrt{\pi} \sigma_{\tau}} \exp \left\{-\left(\frac{\Delta \tau_{u, v}-\mu_{\Delta \tau_{u, v}}}{2 \sigma_{\tau}}\right)^{2}\right\}
$$

where $\Delta \tau_{u, v}=\tau_{u}-\tau_{v}$ and $\mu_{\Delta \tau_{u, v}}=\mu_{\tau_{u}}-\mu_{\tau_{v}}$, with $u \in$ $\{1, \ldots, 4\}$ and $v \in\{0, \ldots, 3\}$.

Given $n$ reflected paths, the number of likelihood functions making up the mixture $\Omega_{n}$ is $\frac{4 !}{(4-n) !}$. As discussed in [8], by construction, the MAL algorithm can not solve transmitter/receiver (Tx/Rx) ambiguity on its own.

2) eMAL policy: The eMAL algorithm tries to improve MAL performances by integrating two more measurements $\alpha_{n, 0}$ and $\beta_{n, 0}$ in the ALFs, as defined in Section III. The main difficulty is that measured $\phi$ can not be a priori distinguished as corresponding to transmitter or receiver (i.e. as $\phi_{t}$ or $\phi_{r}$ ), because of the reciprocity of the propagation channel (i.e. $\mathrm{Tx} / \mathrm{Rx}$ ambiguity). Moreover, as $\theta$ and $\tau$, also $\phi$ measurements suffer from label mapping ambiguity. If the differences (7) for all the $n$ received paths were considered, the algorithm should manage $2^{n+1}$ possibilities due to the $\mathrm{Tx} / \mathrm{Rx}$ ambiguity, which would make $\Omega_{n}$ maximization more complex. Therefore, for each $\Omega_{n}$ component (i.e. paths combination), only one $\alpha$ and $\beta$ is integrated into (12). Indeed, let $\left(q_{1}, \ldots, q_{n}\right)$ be the paths label combination of cardinality $n$ for a given component of $\Omega_{n}$ and $\bar{q}=\min \left\{q_{1}, \ldots, q_{n}\right\}$, then measurements $\alpha_{\bar{q}, 0}$ and $\beta_{\bar{q}, 0}$ are included in the considered $\Omega_{n}$ component.

As measured $\phi$ can not be identified as AoDs or AoAs, the arrays of latter angles corresponding to the path labelled with $\bar{q}$ and to the direct path will be noted $\Phi_{\bar{q}}=\left[\begin{array}{ll}\phi_{\bar{q}, 1} & \phi_{\bar{q}, 2}\end{array}\right]$ and $\Phi_{0}=\left[\begin{array}{ll}\phi_{0,1} & \phi_{0,2}\end{array}\right]$ respectively. Finally, let us define the set of quartets $P$ playing as permutation indexes as follows:

$$
P=\{(1,1,2,2),(1,2,2,1),(2,1,1,2),(2,2,1,1)\}
$$

then, according to the independence hypothesis discussed in Section III, $\Omega_{1, L O S}$ can be written as in (14). Note that eMAL $\Omega_{n}$ are made by $4 \frac{4 !}{(4-n) !}$ terms, which means $2^{2}=4$ times more than corresponding MAL $\Omega_{n}$. Indeed, the uniform probability of $\mathrm{Tx} / \mathrm{Rx}$ role is integrated in eMAL $\Omega_{n}$, in addition to the uniform probability of mapping between detected paths and model paths already present in MAL $\Omega_{n}$. All the $\phi$ permutations (i.e Tx/Rx role) are considered in the eMAL version of $\Omega_{n}$, including the good one and the opposite of the good one, the latter inverting AoDs and AoAs roles, which does not solve Tx/Rx ambiguity as for MAL approach. Nevertheless, the additional measurements are expected to be rewarded with an improvement in estimation performances when a suitable antenna is used.

\section{Remaining Ambiguities}

\section{A. Mirror Ambiguity}

The mirror ambiguity can be defined as the lack of capability in distinguishing the right geometrical configuration from other configurations obtained as mirror images of the right one. In the 2D scenario on Figure 1, the possible mirrors are the axis of $x$ and the axis of $y$. Assuming that static peers do not have an a priori knowledge of the indoor environment (e.g. a map of the building) and are not equipped with absolute reference estimation devices (e.g. [12]), a mirror ambiguity in positioning nodes will always exist whatever the algorithm is (e.g. [4]). This resilient 4-image mirror ambiguity is represented on Figure 2.

\section{B. Transmitter/Receiver Ambiguity}

Given two 2D coordinates, the $\mathrm{Tx} / \mathrm{Rx}$ ambiguity can be defined as the lack of capability in assessing which of the two is the transmitter and which is the receiver. As a possible solution, the $\mathrm{Tx} / \mathrm{Rx}$ ambiguity is expected to be solved out with the mobility of one of the two nodes. Indeed, the spatial coherence between successively observed signals should be the key factor in avoiding Tx/Rx ambiguity. Another possible method could consist in using two different antennas for the two nodes, which should break the symmetry in AoDs and AoAs measurements.

The cumulative effect of mirror and $\mathrm{Tx} / \mathrm{Rx}$ ambiguities makes eight possible configurations not distinguishable one from each other, as each of the four possibilities represented on Figure 2 must be multiplied by two different transmission and receiver roles. 


$$
\begin{aligned}
\Omega_{1, L O S}=\frac{1}{4} f_{D}(d)( & f_{\Theta_{1}}\left(\theta^{(1)}\right) f_{\Delta T_{1,0}}\left(\Delta \tau^{(1,0)}\right) \sum_{\left(i_{1}, i_{2}, i_{3}, i_{4}\right) \in P} f_{A_{1,0}}\left(\phi_{(1), i_{1}}-\phi_{(0), i_{2}}\right) f_{B_{1,0}}\left(\phi_{(1), i_{3}}-\phi_{(0), i_{4}}\right)+ \\
& f_{\Theta_{2}}\left(\theta^{(1)}\right) f_{\Delta T_{2}, 0}\left(\Delta \tau^{(1,0)}\right) \sum_{\left(i_{1}, i_{2}, i_{3}, i_{4}\right) \in P} f_{A_{2,0}}\left(\phi_{(1), i_{1}}-\phi_{(0), i_{2}}\right) f_{B_{2}, 0}\left(\phi_{(1), i_{3}}-\phi_{(0), i_{4}}\right)+ \\
& f_{\Theta_{3}}\left(\theta^{(1)}\right) f_{\Delta T_{3,0}}\left(\Delta \tau^{(1,0)}\right) \sum_{\left(i_{1}, i_{2}, i_{3}, i_{4}\right) \in P} f_{A_{3,0}}\left(\phi_{(1), i_{1}}-\phi_{(0), i_{2}}\right) f_{B_{3,0}}\left(\phi_{(1), i_{3}}-\phi_{(0), i_{4}}\right)+ \\
& \left.f_{\Theta_{4}}\left(\theta^{(1)}\right) f_{\Delta T_{4,0}}\left(\Delta \tau^{(1,0)}\right) \sum_{\left(i_{1}, i_{2}, i_{3}, i_{4}\right) \in P} f_{A_{4,0}}\left(\phi_{(1), i_{1}}-\phi_{(0), i_{2}}\right) f_{B_{4,0}}\left(\phi_{(1), i_{3}}-\phi_{(0), i_{4}}\right)\right)
\end{aligned}
$$
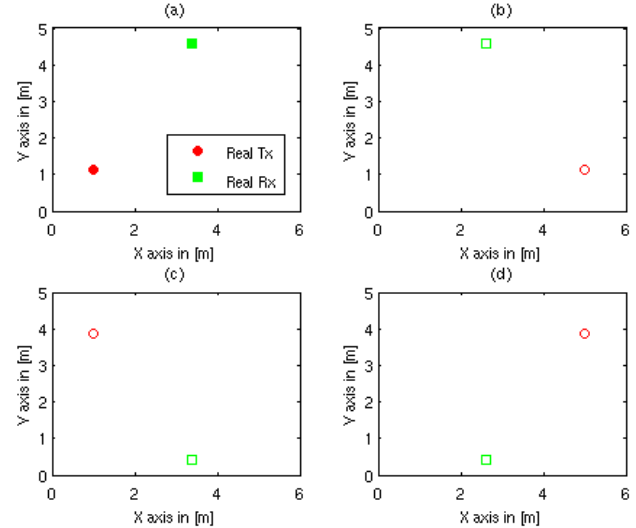

Fig. 2. Real configuration (a) and mirror images (b), (c) and (d)

\section{ANTENNAS}

Both described algorithms rely on the capability of locally reproducing the expected received waveforms but eMAL also accounts for the radiation spatial diversity of antennas in order to estimate AoDs and AoAs. Several works (e.g. [13], [14]) investigated on appropriate UWB antenna indicators. Two of them seem to critically impact the performances of the described algorithms. Indeed, the space diversity of UWB antennas has been quantified in a distortion factor that takes into account the cross-correlation between transmitted/received pulses, as a function of the radiation directions. Moreover, the Mean Realized Gain (MRG) that is the antenna gain integrated over the signal bandwidth, should be constant with respect to radiating directions. Indeed, if the overall gain for each direction is almost constant, all paths are expected to be received.

\section{A. Conical Monopole Antenna}

The Conical Monopole Antenna (CMA) 118/A is the antenna shown on Figure 3. The omni-directional measured radiation in azimuth, reported on Figure 4, is guaranteed over the whole decade $[1,10] \mathrm{GHz}$ thanks to its cylindrical symmetry structure. This UWB antenna provides a very low pulse distortion that is the same in all the horizontal angular directions. Consequently, the main application of CMA 118/A is the channel sounding (e.g. [15], [16]). Indeed, CMA dimensions and weight (i.e. about $1 \mathrm{~kg}$ [9]) discourage its use in the context of WSNs.

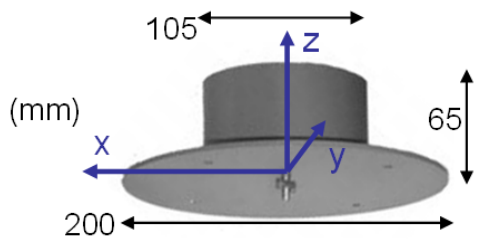

Fig. 3. Conical Monopole antenna 118/A

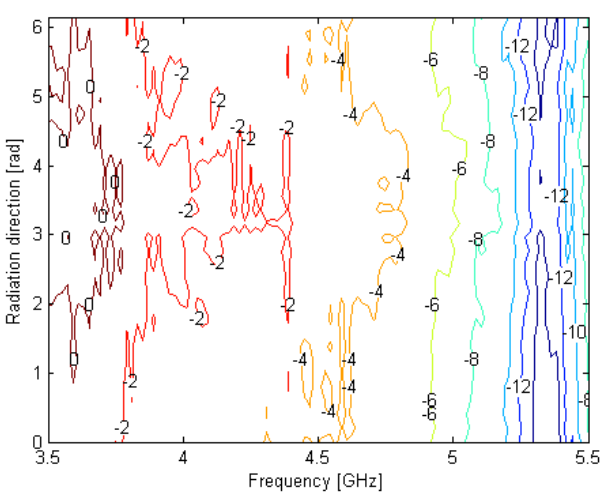

Fig. 4. CMA 118/A gain in azimuth plane in $\mathrm{dB}$

\section{B. Middle Alva Antenna}

The Middle ALVA (MA) antenna illustrated on Figure 5 is a compact UWB antenna derived from [17] for Body Area Network (BAN) applications (e.g. [18]). This low cost, light weight (i.e. about $30 \mathrm{~g}$ ) miniature antenna can be seen either as a short circuited printed folded dipole or as a notch antenna. The notch design has been modified in order to achieve wideband behavior with the elliptical shape of the dipole arms. As a consequence, the wideband impedance matching is obtained by combining two different antenna modes. In the band $[3.5,5.5] \mathrm{GHz}$, the notch acts as an aperture antenna with small directive characteristics. The antenna gain obtained by simulation in azimuth plane is shown on Figure 6.

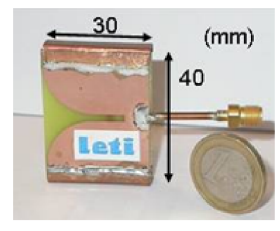

Fig. 5. Middle Alva antenna

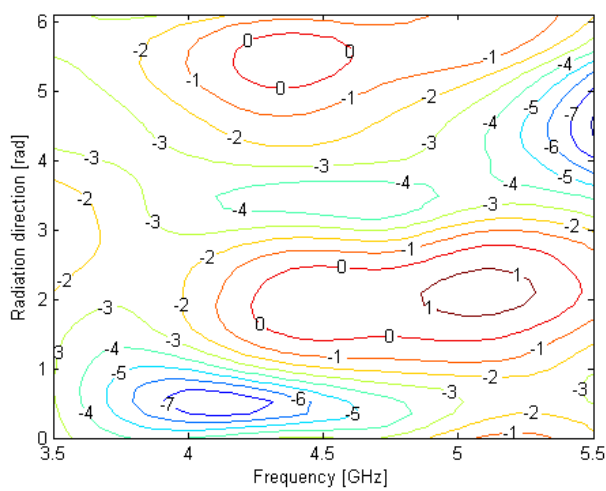

Fig. 6. MA gain in azimuth plane in $\mathrm{dB}$ 


\section{Monopolar Wire Patch Antenna}

The Monopolar Wire-Patch (MWP) (e.g. [15]) is an UWB antenna with low profile design, whose dimensions are depicted on Figure 7 and which weights about $300 \mathrm{~g}$. The antenna is well matched between 3 and $10 \mathrm{GHz}$ and is mostly intended for indoor high data rate communications. The antenna radiation is quasi omni-directional in the azimuth plane, despite the non cylindrical symmetry of the monopole. A dipole-like pattern is stable over the first octave as the ground plane is still electrically small. Beyond $5 \mathrm{GHz}$, the omni-directionality in azimuth is slightly degraded due to the non cylindrical symmetry of the monopole. The antenna gain in azimuth plane obtained by simulation is depicted on Figure 8 .

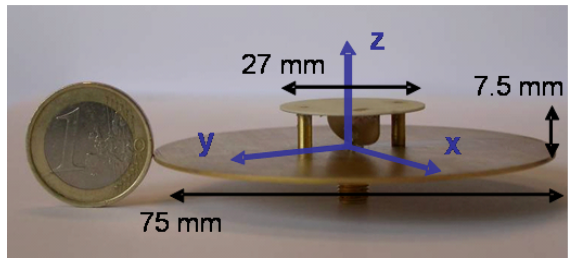

Fig. 7. Monopolar Wire-Patch (MWP) antenna

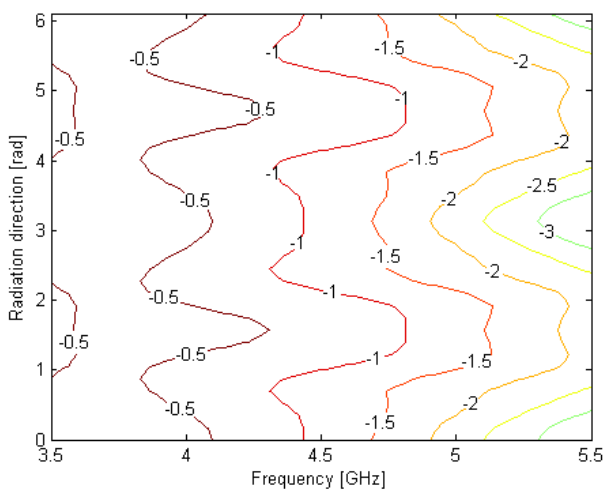

Fig. 8. MWP gain in azimuth plane in $\mathrm{dB}$

\section{Simulation Results}

The geometrical scenario associated with shown results is the same as that described in [8]. As an example, on Figure 9 an observation of the received noisy signal is provided for CMA, MA and MWP antennas. According to the LOS hypothesis, the first received path is forced to be estimated as a direct path. Only two types of interactions are available in the local waveforms database: direct path (i.e. free space propagation) and reflection on brick.

The Power Spectral Density (PSD) of the transmitted signal complies with the FCC UWB mask (e.g. [2]). Moreover, as in [19], the simulated system employs a Pulse Repetition Period (PRP) of $100 \mathrm{~ns}$ and the signal has a bandwidth of $2 \mathrm{GHz}$ at $-10 \mathrm{~dB}$ of the PSD maximum, centered around $4.5 \mathrm{GHz}$. The received signal is affected by thermal noise (i.e. Gaussian distributed, $N_{0}=-174 \mathrm{dBm} / \mathrm{Hz}$ ) filtered in the signal band and a noise figure of $10 \mathrm{~dB}$ accounts for a more realistic receiver (e.g. [2]). The sampling frequency for the received signal is assumed to be $15 \mathrm{GHz}$, beyond Nyquist frequency.

According to simulation results, the best trade-off between execution time and estimation performances for the presented algorithms is obtained with the thresholds represented on Table
TABLE I

EMAL THRESHOLDS

\begin{tabular}{r||c|c|c}
\hline \hline & $\xi_{C C}$ & $\delta_{L T, 1}$ & $\delta_{L T, 5}$ \\
\hline \hline CMA & 0.20 & 0.70 & 0.99 \\
MA & 0 & 0.80 & 0.99 \\
MWP & 0.20 & 0.85 & 0.95 \\
\hline
\end{tabular}

TABLE II

MAL THRESHOLDS

\begin{tabular}{r||c|c|c}
\hline \hline & $\xi_{C C}$ & $\delta_{L T, 1}$ & $\delta_{L T, 5}$ \\
\hline \hline CMA & 0.15 & 0.20 & 0.95 \\
MA & 0 & 0.20 & 0.95 \\
MWP & 0.15 & 0.25 & 0.95 \\
\hline \hline
\end{tabular}

I and II. The shown results have been obtained with this algorithm calibration. In addition, the standard deviations considered for the averaged likelihood computation are presented on Table III.

Positioning results for CMA, MA and MWP antennas are respectively on Figure 10,11 and 12. For the CMA antenna, eMAL and MAL algorithms provide approximately the same estimation error, which is lower than $0.6 \mathrm{~m}$ for $90 \%$ of estimates and with a median value of about $0.55 \mathrm{~m}$, as illustrated on Figure 10. On the contrary, a strong difference in positioning errors can be found between eMAL and MAL algorithms applied to MA antenna. Indeed, the positioning error obtained with the extended algorithm is lower than 0.6 $\mathrm{m}$ for $90 \%$ of estimates but with a median value of about $0.04 \mathrm{~m}$, as illustrated on Figure 11a. Finally, the positioning error Cumulative Density Functions (CDFs) obtained with a MWP antenna are presented on Figure 12, highlighting a slight improvement obtained using eMAL in comparison with MAL.

These positioning results clearly show the tight relationship between antenna deformation properties and algorithm measurements. Accordingly, the antennas characterized by almost perfect omni-directional radiation pattern (e.g. CMA and MWP) can not take advantage of additional AoAs and AoDs information. Otherwise, the radiation diversity of MA antenna allows eMAL to better estimate nodes positions in comparison with MAL. The different reliability of measurements with respect to a given antenna is also illustrated by standard deviations on Table III. Therefore, the positioning estimation exploiting only AoIs and ToAs (i.e. with MAL algorithm, on Figure 11b) can not be satisfactorily achieved using MA antenna but it becomes possible, on the contrary, when AoD and AoA measurements are integrated, as illustrated on Figure 11a. The antinomy of radiation diversity and proneness of radiated waveforms to be signed by the AoI was hypothesized in [20] and seems to be validated by the discussed results. It

TABLE III

STANDARD DEVIATIONS USED FOR EMAL AND MAL APPROACH

\begin{tabular}{r||c|c|c||c|c}
\hline \hline \multicolumn{1}{c||}{} & \multicolumn{3}{c||}{ eMAL } & \multicolumn{2}{c}{ MAL } \\
\cline { 2 - 6 } & $\sigma_{\theta}[\mathrm{deg}]$ & $\sigma_{\tau}[\mathrm{ns}]$ & $\sigma_{\phi}[\mathrm{deg}]$ & $\sigma_{\theta}[\mathrm{deg}]$ & $\sigma_{\tau}[\mathrm{ns}]$ \\
\hline \hline CMA & 9 & 0.25 & 23 & 9 & 0.25 \\
MA & 18 & 0.125 & 15 & 9 & 0.125 \\
MWP & 9 & 0.125 & 20 & 9 & 0.125 \\
\hline \hline
\end{tabular}


is also interesting to note that positioning error CDFs seems to be stepwise, particularly these corresponding to MA and MWP antennas. This shape is due to the presence of local maxima in the ALFs, which makes ALFs maximization not always correct. Therefore, bad positioning solutions are not continuous but discrete and corresponding to high but local maxima.

Finally, the CDFs of room dimension $D_{x}$ estimation errors expressed in percentage are displayed on Figure 13, 14 and 15 for CMA, MA and MWP antennas respectively. As for the positioning error, eMAL and MAL algorithms perform almost the same if a CMA or a MWP antenna is employed. On the contrary, a sharp improvement with respect to MAL algorithm can be observed using eMAL algorithm with MA, as the extended algorithm shows an error lower than $0.8 \%$ for $90 \%$ of estimates. Nevertheless, the observed $D_{x}$ errors can be generally considered very interesting for all the three antennas, as the error is always lower than $2.5 \%$, whatever the algorithm and the antenna are.

\section{CONCLUSION}

This paper has compared the Maximum Averaged Likelihood (MAL) algorithm and its extension eMAL using three realistic antennas (i.e. Conical Monopole, Middle Alva and Monopolar Wire Patch). Both algorithms exploit the maximum averaged likelihood concept, using Angles of Incidence (AoIs) and Times of Arrival (ToAs) as measurements for MAL but Angles of Arrival (AoAs), Angles of Departure (AoDs), AoIs and ToAs measurements for eMAL. Simulations showed that the eMAL algorithm applied to a light, small and low power antenna like the Middle Alva can provide a positioning error lower than $0.6 \mathrm{~m}$ for $90 \%$ of estimates and even a median error of $0.04 \mathrm{~m}$, after removing mirror and $\mathrm{Tx} / \mathrm{Rx}$ ambiguities. Moreover, an error on room dimension lower than $0.8 \%$ for $90 \%$ of estimates is an acquired result for the Middle Alva in the chosen framework. Considering the context of use of the latter antenna, this main result may disclose new ways for BAN opportunistic localization. Even if a simplified scenario has been considered, it seems important to recall that the IR-UWB pair is static and that both algorithms do not need anchors, which makes obtained results potentially interesting.

Future works will investigate performances with relaxed technological constraints (e.g. lower frequency sampling) and introducing mobility.

\section{ACKNOWLEDGEMENT}

This work has been partly carried out in the frame of the EUWB European Project [21].

\section{REFERENCES}

[1] D. Cook and S. Das, Smart Environments: Technology, Protocols and Applications, Wiley-Interscience, November 2004.

[2] T. Kaiser and M.-G. Di Benedetto, UWB communication systems, Hindawi Publishing Corporation, 2006.

[3] S. Gezici, Z. Tian, G.B. Giannakis, et al., "Localization via ultrawideband radios: a look at positioning aspects for future sensor networks", IEEE Signal Processing Magazine, vol. 22, no. 4, pp. 70-84, 2005.

[4] W. Guo, N.P. Filer, and S.K. Barton, "2D indoor mapping and locationsensing using an impulse radio network", in Proc. IEEE ICUWB'05, Zurich, Switzerland, September 2005, pp. 296-301.

[5] R. Zetik, J. Sachs, and R. Thoma, "Modified cross-correlation back projection for UWB imaging: numerical examples", in Proc. IEEE ICUWB'05, Zurich, Switzerland, September 2005, p. 5.

[6] J. Seitz, M. Schaub, O. Hirsch, et al., "UWB feature localization for imaging", in Proc. IEEE ICUWB'08, Hannover, Germany, September 2008, vol. 2, pp. 199-202.
[7] H.J. Chang, C.S.G. Lee, Lu Yung-Hsiang, and Y.C. Hu, "P-SLAM: simultaneous localization and mapping with Environmental-Structure prediction", IEEE Trans. on Robotics, vol. 23, no. 2, pp. 281-293, April 2007.

[8] V. La Tosa, B. Denis, and B. Uguen, "Maximum averaged likelihood estimation tree for Anchor-Less localization exploiting IR-UWB multipaths", in Proc. IEEE VTC Spring 2010, Taipei, Taiwan, May 2010.

[9] "Antenna research associates (ARA)", http://www.ara-inc.com.

[10] F. Tchoffo-Talom, B. Uguen, E. Plouhinec, and G. Chassay, "A sitespecific tool for UWB channel modeling", in Proc. Joint UWBST \& IWUWBS, Kyoto, Japan, May 2004, pp. 61-65.

[11] V. La Tosa, B. Denis, and B. Uguen, "A preliminary investigation on angular parameters estimation in a simplified IR-UWB indoor multipath scenario", in Proc. IEEE WCNC'09, Budapest, Hungary, April 2009, pp. 1-6.

[12] B. Bahreyni and C. Shafai, "A resonant micromachined magnetic field sensor", IEEE Sensors Journal, vol. 7, no. 9, pp. 1326-1334, 2007.

[13] O.E. Allen, D.A. Hill, and A.R. Ondrejka, "Time-domain antenna characterizations", IEEE Trans. on Electromagnetic Compatibility, vol. 35, no. 3, pp. 339-346, 1993.

[14] C. Roblin, "Ultra compressed parametric modeling for symmetric or pseudo-symmetric UWB antenna", in Proc. IEEE ICUWB'08, Hannover, Germany, September 2008, vol. 2, pp. 109-112.

[15] J. Keignart and N. Daniele, "Subnanosecond UWB channel sounding in frequency and temporal domain", in Proc. IEEE UWBST 2002, Baltimore, USA, May 2002, pp. 25-30.

[16] P. Pagani and P. Pajusco, "Experimental analysis of the ultra wideband propagation channel over the $3.1 \mathrm{GHz}-10.6 \mathrm{GHz}$ frequency band", in Proc. IEEE PIMRC'06, Helsinki, Finland, September 2006, pp. 1-5.

[17] F. Demeestere, C. Delaveaud, and J. Keignart, "A compact UWB antenna with a wide band circuit model and a time domain characterization", in Proc. IEEE ICUWB'06, Waltham, USA, 2006, pp. 345-350.

[18] D'Errico R. and Ouvry L., "Time-variant BAN channel characterization”, in Proc. IEEE PIMRC'09, Tokyo, Japan, September 2009.

[19] M. Navarro, S. Prior, and M. Najar, "Low complexity frequency domain TOA estimation for IR-UWB communications", in Proc. IEEE VTC2006 Fall, Montreal, Canada, September 2006, pp. 1-5.

[20] V. La Tosa, B. Denis, F. Tchoffo-Talom, and B. Uguen, "Joint directions finding and material typecasting through IR-UWB communications", in Proc. IEEE PIMRC'08, Cannes, France, September 2008, pp. 1-6.

[21] "EUWB, ICT-Project (Contract number 215669)", http://www.euwb.eu/.
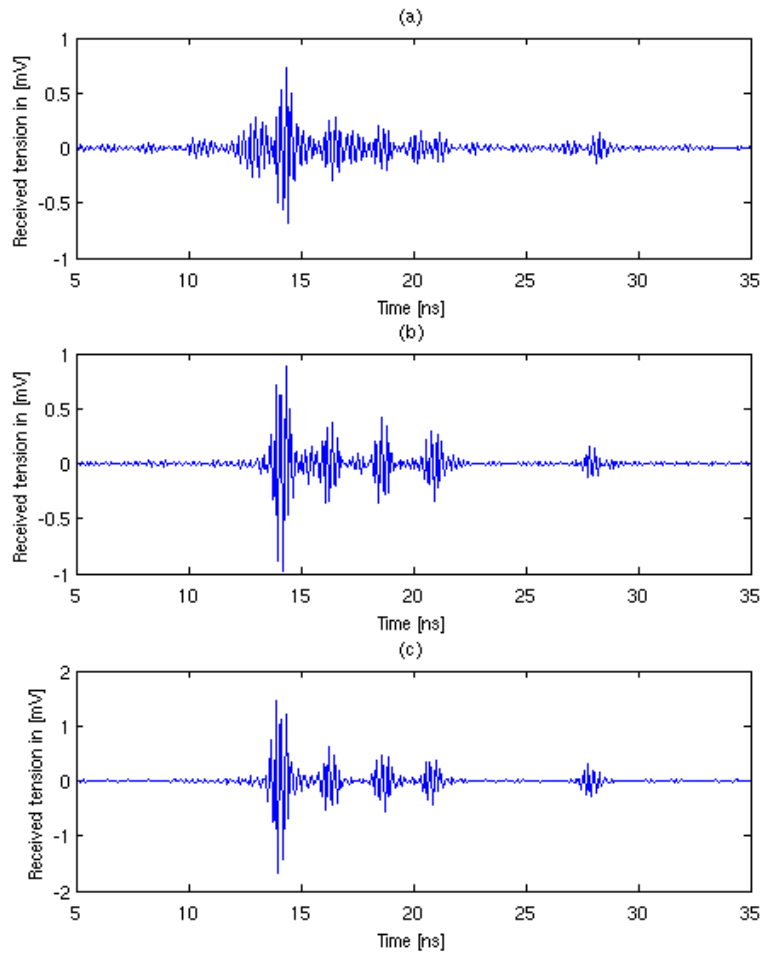

Fig. 9. Noisy received signal obtained by Ray Tracing simulation using a Conical Monopole (a), Middle Alva (b) and Monopolar Wire-Patch antennas 

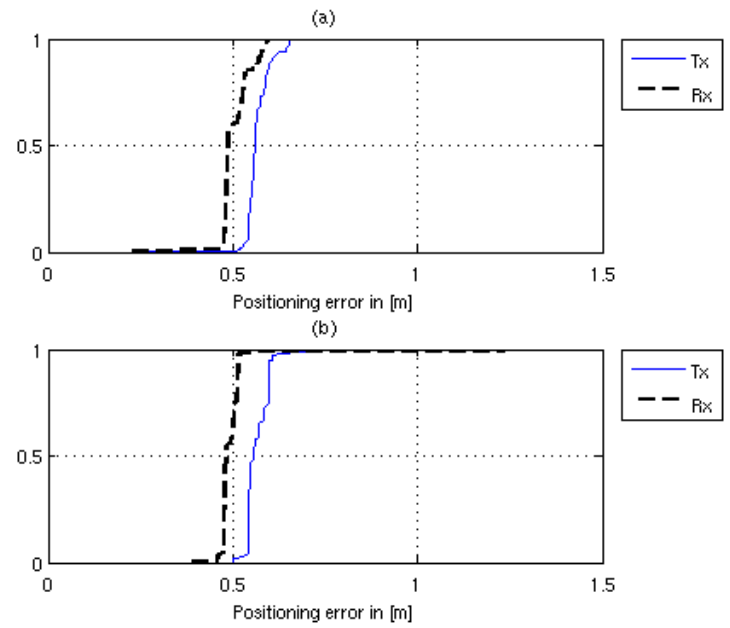

\begin{tabular}{|l|}
\hline$-{ }^{\mathrm{TX}}$ \\
\hline$-{ }_{\mathrm{Rx}}$ \\
\hline
\end{tabular}

Fig. 10. Positioning error CDFs using Conical Monopole antennas, after ambiguities solving, eMAL (a) and MAL (b) algorithm
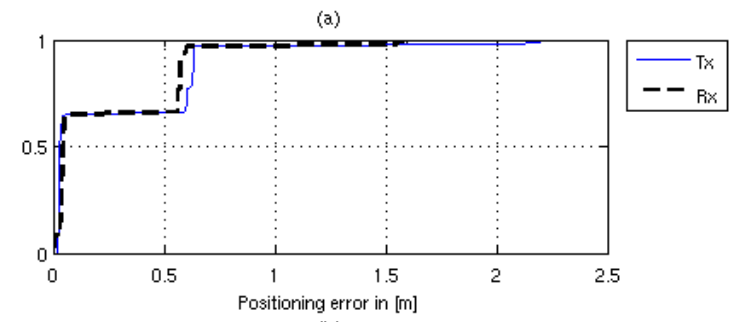

(b)
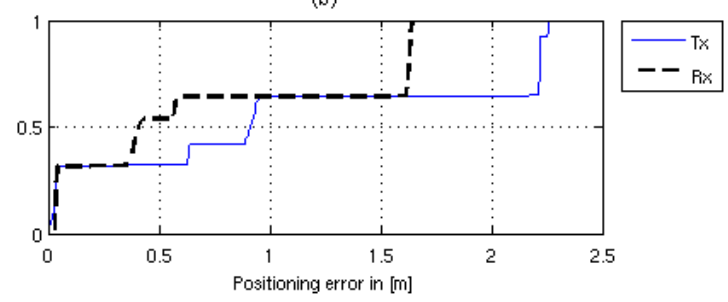

Fig. 11. Positioning error CDFs using Middle Alva antennas, after ambiguities solving, eMAL (a) and MAL (b) algorithm
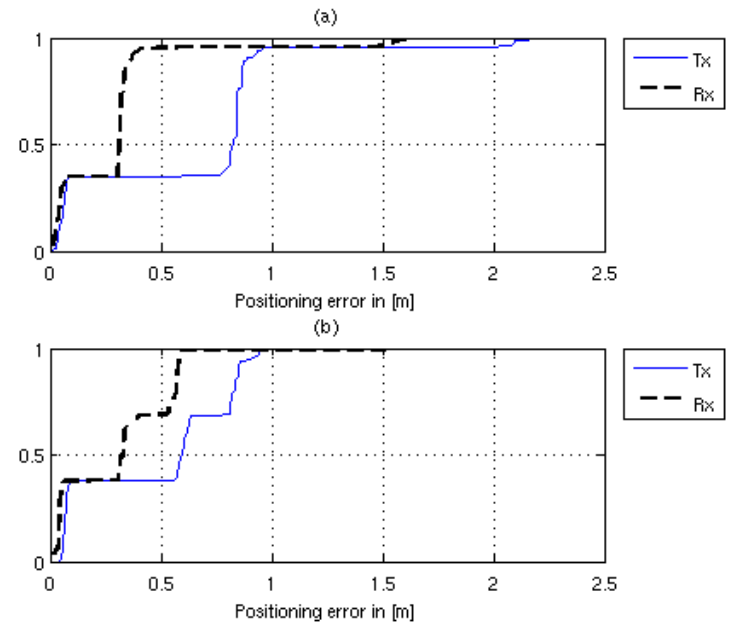

Fig. 12. Positioning error CDFs using Monopole Wire-Patch antennas, after ambiguities solving, eMAL (a) and MAL (b) algorithm

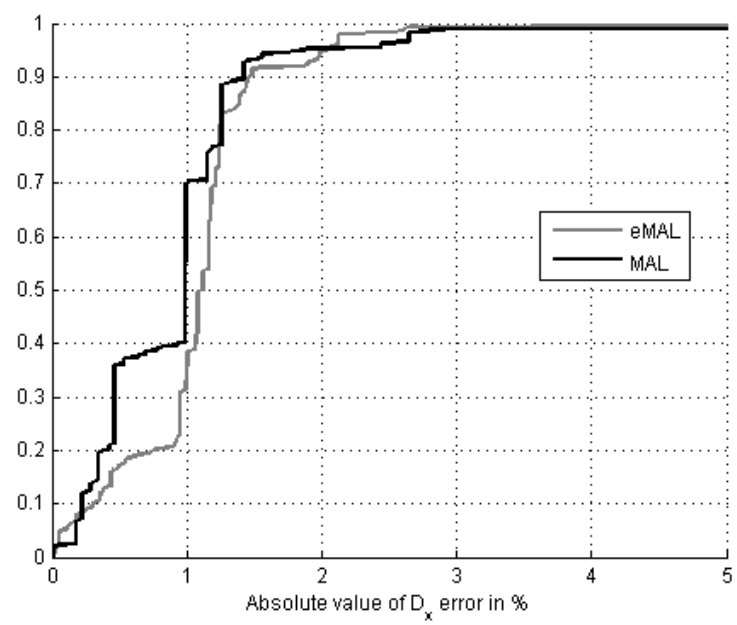

Fig. 13. $D_{x}$ error CDFs expressed in percentage, using Conical Monopole antennas, eMAL and MAL algorithm

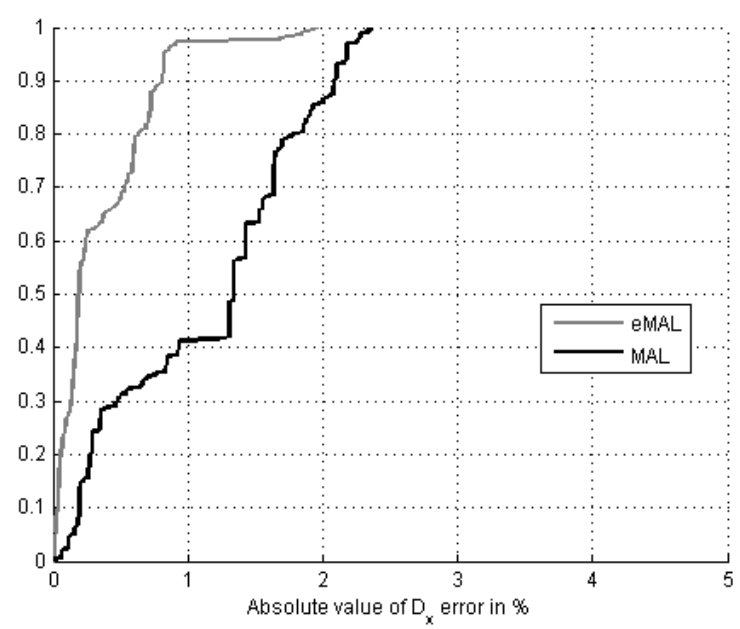

Fig. 14. $D_{x}$ error CDFs expressed in percentage, using Middle Alva antennas, eMAL and MAL algorithm

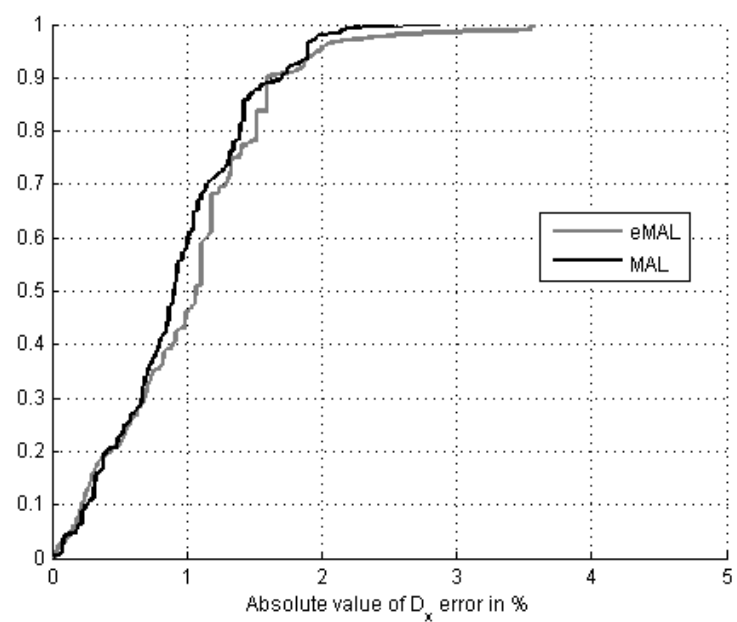

Fig. 15. $D_{x}$ error CDFs expressed in percentage, using Monopole Wire-Patch antennas, eMAL and MAL algorithm 